\title{
Production of Stealthin C Involves an S-N-Type Smiles Rearrangement
}

\section{Citation}

Wang, Peng, Gloria J. Hong, Matthew R. Wilson, and Emily P. Balskus. 2017. "Production of Stealthin C Involves an S-N-Type Smiles Rearrangement." Journal of the American Chemical Society 139 (8) (February 16): 2864-2867. doi:10.1021/jacs.6b10586.

\section{Published Version}

10.1021/jacs.6b10586

\section{Permanent link}

http://nrs.harvard.edu/urn-3:HUL.InstRepos:32749937

\section{Terms of Use}

This article was downloaded from Harvard University's DASH repository, and is made available under the terms and conditions applicable to Other Posted Material, as set forth at http:// nrs.harvard.edu/urn-3:HUL.InstRepos:dash.current.terms-of-use\#LAA

\section{Share Your Story}

The Harvard community has made this article openly available.

Please share how this access benefits you. Submit a story.

Accessibility 


\title{
Production of stealthin C involves an S-N-type Smiles rearrangement
}

\author{
Peng Wang, Gloria J. Hong, Matthew R. Wilson, and Emily P. Balskus* \\ Department of Chemistry \& Chemical Biology, Harvard University, 12 Oxford St, Cambridge, Massachusetts 02138, United \\ States.
}

Supporting Information Placeholder

\begin{abstract}
The kinamycin family of aromatic polyketide natural products contains an atypical angucycline ring system substituted with a diazo group. The enzymatic chemistry involved in constructing both of these structural features has been largely unexplored. Here we report the in vivo and in vitro production of seongomycin, a shunt product from this pathway, and stealthin $\mathrm{C}$, a proposed biosynthetic precursor to the kinamycins. We show that a single enzyme, the flavin-dependent monooxygenase AlpJ, can generate these metabolites from $\mathrm{N}$-acetyl-L-cysteine and Lcysteine, respectively, and that the synthesis of stealthin $\mathrm{C}$ likely proceeds via a non-enzymatic S-N-type Smiles rearrangement. This unexpected route to stealthin $\mathrm{C}$ reveals a distinct approach to install aromatic amino groups in metabolites and raises questions about the intermediacy of this species in kinamycin production.
\end{abstract}

The diazofluorenes are a group of DNA-damaging natural products produced by both soil and marine Actinomycetes (Figure 1A). ${ }^{1,2}$ Family members, including the kinamycins and the lomaiviticins, share a common diazotetrahydrobenzo $[b]$ fluorene core scaffold that has been a rich source of inspiration for synthetic chemists and chemical biologists who have pursued syntheses of the natural products as well as simplified analogs for biological evaluation. ${ }^{1}$

Despite intense interest in these molecules, our understanding of diazofluorene biosynthesis is largely limited to in vivo experiments involving the kinamycins, monomeric metabolites produced by Streptomyces murayamaensis and Streptomyces ambofaciens. ${ }^{3}$ Gould and co-workers identified additional metabolites from $S$. murayamaensis, including the aromatic polyketide dehydrorabelomycin (1). $\mathbf{1}$ is a key intermediate in the production of all angucycline polyketides, including the atypical angucyclines gilvocarcin and jadomycin. ${ }^{4}$ Feeding deuterated 1 to $S$. murayamaensis confirmed its connection to kinamycin $\mathrm{D}$, suggesting a biosynthetic route involving $\mathrm{C}$-ring contraction and nitrogen insertion events (Figure 1B). ${ }^{5}$

Seongomycin (2), stealthin C (3), and kinobscurinone (4) are kinamycin-related metabolites isolated from $S$. murayamaensis mutants generated via chemical mutagenesis. ${ }^{6}$ Feeding studies implicated both $\mathbf{3}$ and $\mathbf{4}$ as biosynthetic intermediates (Figure 1B) ${ }^{6 a, b}$ while 2 and its counterpart from lomaiviticin biosynthesis (homoseongomycin) were proposed to be shunt products derived from the reaction of the diazo-containing metabolites prekinamycin and homoprekinamycin with cellular thiols. ${ }^{7}$ Notably, 2-4 were produced upon heterologous expression of a partial kinamycin biosynthetic gene cluster. ${ }^{8}$

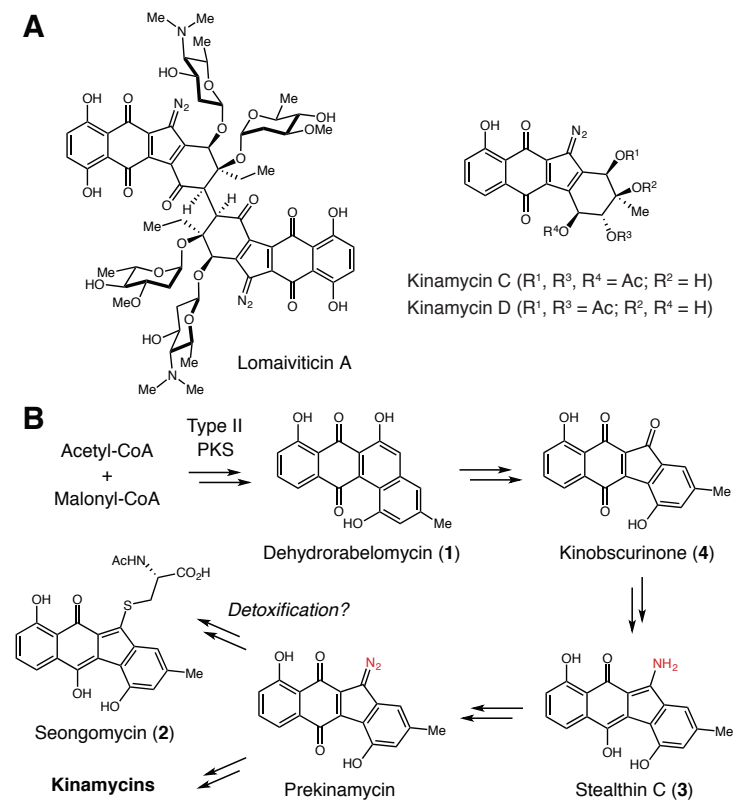

Figure 1. Biosynthesis of the diazofluorene natural products. A) Representative diazofluorene natural products. B) Proposed biosynthetic pathway to the kinamycins and seongomycin.

These initial studies left many biosynthetic questions unanswered, including the identities of the enzymes involved in ben$\mathrm{zo}[b]$ fluorene ring formation and diazo generation. The discovery of the complete kinamycin and lomaiviticin biosynthetic gene clusters has provided new opportunities to investigate these intriguing transformations. ${ }^{3 \mathrm{c}, 9}$ Here we report the in vivo and in vitro characterization of enzymatic machinery capable of synthesizing 2 and 3 from 1 and either $N$-acetyl-L-cysteine or L-cysteine. Assays with labeled substrates and substrate analogs suggest that formation of 3 involves a non-enzymatic S-N-type Smiles rearrangement. This discovery calls into question the proposed role of stealthin $\mathrm{C}$ as a biosynthetic intermediate.

We began by identifying the enzymes involved in generating the benzo $[b]$ fluorene ring system. Yang and co-workers reported two oxidases from $S$. ambofaciens $(\mathrm{AlpJ} / \mathrm{K}$ ) that produce $\mathbf{2}$ from $\mathbf{1}$ in vivo and in vitro. ${ }^{10}$ Simultaneously, we pursued a similar line of inquiry that revealed these enzymes are also capable of producing proposed biosynthetic intermediate 3 in vivo and in vitro. We uncovered candidate benzo[b]fluorene ring-forming enzymes by comparing the kin and lom biosynthetic gene clusters to gene clusters that produce other angucycline polyketides. ${ }^{11}$ In the jadomycin and gilvocarcin pathways, flavin dependent monooxygenases JadG and GilOII perform a Baeyer-Villiger-type oxida- 
tive $\mathrm{C}-\mathrm{C}$ bond cleavage reaction. ${ }^{4} \mathrm{We}$ located homologous flavindependent monooxygenases encoded in the kin and lom clusters (AlpJ/KinG and Lom28). These JadG homologs were always colocalized with a second flavin-dependent oxidase (AlpK/KinO1 and Lom27) that was absent from the gil and jad gene clusters (Figure S1), suggesting that this enzyme might also play a role in benzo $[b]$ fluorene formation.

To initiate our studies, we cloned alpJ and alpK from S. ambofaciens into the Streptomyces expression vector pUWL201PW in an operon under the control of the $\operatorname{erm} E^{*}$ promoter. The resulting plasmid pAlpJK was transformed into Streptomyces lividans TK64 to afford recombinant strain pAlpJK/TK64. Feeding 1 to pAlpJK/TK64 led to production of $\mathbf{2}\left(\mathrm{m} / \mathrm{z}[\mathrm{M}+\mathrm{H}]^{+}=454.0960\right)$ (Figures 2 and S2), confirming that the AlpJK enzyme pair catalyzed the $\mathrm{C}-\mathrm{C}$ bond cleavage and ring contraction reactions.
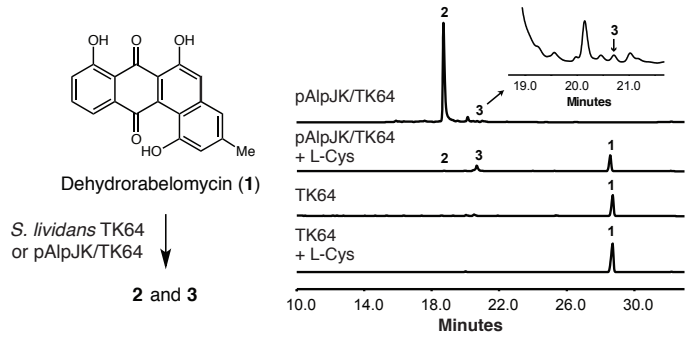

Figure 2. AlpJ converts dehydrorabelomycin to seongomycin and stealthin $\mathrm{C}$ in vivo. HPLC analysis of culture extracts.

Interestingly, we also detected a minor metabolite that had a similar UV-visible absorption spectrum to stealthin C (3) $(\mathrm{m} / z$ [M $+\mathrm{H}^{+}=308.0924$ ) (Figures 2 and S3) ${ }^{6 \mathrm{~b}}$ This observation was unexpected as aminobenzo $[b]$ fluorene formation is proposed to require at least one additional enzyme for nitrogen transfer. ${ }^{6 b}$ Though this result suggested that AlpJK could generate 3 in vivo, the yield of this metabolite was extremely low compared to that of 2. This difference could arise from the availability of biosynthetic precursors to these metabolites. The $\mathrm{N}$-acetyl-cysteine group of $\mathbf{2}$ is likely derived from mycothiol, which is present at high concentrations in Streptomyces lividans cells $(\sim 2.2 \mathrm{mM}) .{ }^{12}$ We wondered if the amino group of $\mathbf{3}$ might derive from L-cysteine (present at $\sim 0.2 \mathrm{mM}) .{ }^{12}$ We fed 1 and L-cysteine $(\sim 11 \mathrm{mM})$ to pAlpJK/TK64 and observed both a loss of $\mathbf{2}$ and an 11-fold increase in $\mathbf{3}$ (Figure 2). Feeding 1 and ${ }^{15} \mathrm{~N}$-labeled L-cysteine confirmed that the nitrogen atom of this amino acid is incorporated into stealthin C (Figure S4).

To explore these processes in vitro, AlpJ and the AlpK homo$\log \mathrm{KinO} 1$ were expressed in E. coli (Figure S5). While purified AlpJ was colorless, purified KinO1 was bright yellow. LC-MS analysis of the supernatants of both enzymes revealed FAD (Figures S6 and S7). When 1 was incubated with AlpJ, the E. coli FAD reductase Fre, ${ }^{13}$ FAD, and NADPH, we observed a new peak by HPLC that did not correspond to the hydroxyoxepinone intermediate noted previously in in vitro assays with $\mathbf{1}$ and GilOII or $\mathrm{JadG}^{4 \mathrm{~d}}{ }^{\mathrm{d}}$ Subsequent LC-MS analysis indicated that this new compound had $\mathrm{m} / \mathrm{z}[\mathrm{M}-\mathrm{H}]^{-}=581.1218$ (Figure S8, trace ii and Figure S9), which corresponds to a dimeric benzofluorene (5). This product was also identified by Yang and co-workers. ${ }^{10}$ When we replaced Fre with KinO1, the consumption of 1 was slower and $\mathbf{5}$ was not detected (Figure S8, trace iii). Furthermore, AlpJ alone generated $\mathbf{5}$ with reduced efficiency (Figure S8, trace i). These results show that AlpJ can catalyze both the oxidative $\mathrm{C}-\mathrm{C}$ bond cleavage reaction and the subsequent ring-closing reaction. The altered product distribution in the presence of KinO1 implies this enzyme both reduces FAD and works as an ancillary oxygenase to enhance the production of hydroquinone-kinobscurinone and avoid $\mathbf{5}$, a function also proposed by Yang and co-workers. ${ }^{10}$
We next added $N$-acetyl-L-cysteine to the HPLC assays described above and observed efficient formation of a new product with a mass corresponding to 2 (Figures S10 and S11). AlpJ produced 2 both in the presence and absence of KinO1/Fre, although the qualitative rate of production was lower without a partner oxidoreductase. The dispensability of KinO1/Fre suggests a supportive but nonessential role in the formation of 2 in vitro. This contrasts with the results of Yang and co-workers.

The discovery that $\mathbf{2}$ can be accessed directly from 1 revises our thinking about its origins. Our work, and that of Yang and coworkers, demonstrates an alternate route to $\mathbf{2}$ that does not require a diazo-containing metabolite. We propose that AlpJ first catalyzes the ring opening reaction via a Baeyer-Villiger oxidation mechanism, giving rise to a previously posited aldehyde/acid intermediate (Scheme S1). ${ }^{4 a, b}$ This species may then undergo ring closure, decarboxylation, and dehydration to give the benzofluorene ring. The $\mathrm{C}-5$ position of benzofluorene could be further oxidized to give hydroquinone-kinobscurinone, which could be attacked by the thiol of $N$-acetyl-cysteine, affording $\mathbf{2}$.

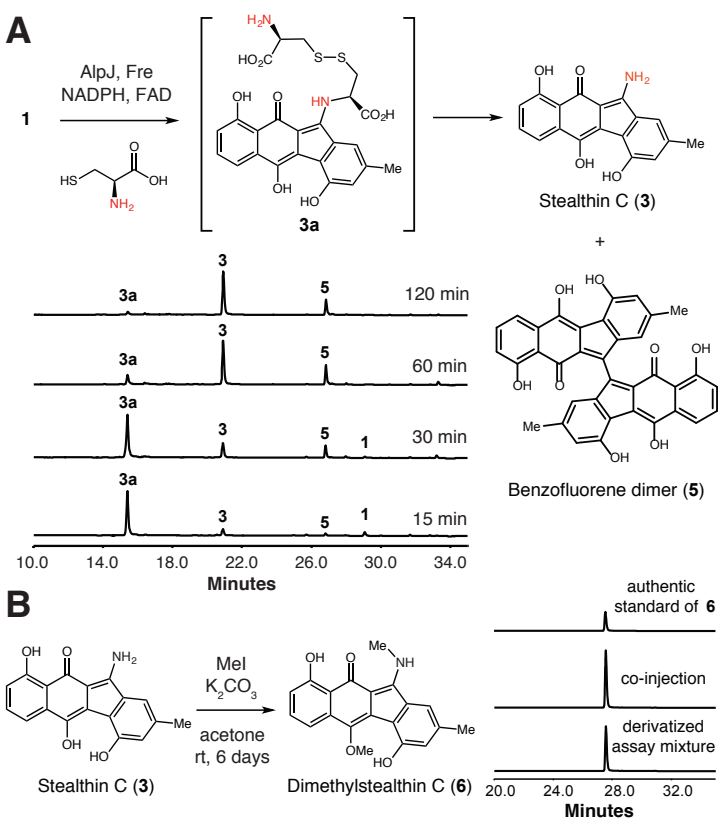

Figure 3. AlpJ converts 1 and L-cysteine to stealthin C (3). A) HPLC analysis of in vitro assays containing AlpJ and Fre. $5=$ diazofluorene dimer. Additional control assays are shown in Figures S16 and S17. B) Derivatization of AlpJ assay product 3.

We then explored the reactivity of AlpJ and Fre toward $\mathbf{1}$ and L-cysteine in vitro. We observed the formation of two new peaks that displayed UV-vis absorbances similar to that of $\mathbf{3}$ (Figures $\mathrm{S} 12$ trace ii, S13, and S14). An assay time course suggested that one of these products (3a) was a reaction intermediate, as its depletion accompanied the formation of the second product (3) (Figure 3A). The two products were also generated by AlpJ and KinO1 (Figure S12, trace iii). Isolation and high-resolution mass spectrometry (HRMS) analysis of the final product revealed $\mathrm{m} / \mathrm{z}$ $[\mathrm{M}+\mathrm{H}]^{+}=308.0929$, which matches the molecular formula of $\mathbf{3}$ The characterization of this product was complicated by the fact that $\mathbf{3}$ is NMR-silent. ${ }^{14}$ We therefore derivatized $\mathbf{3}$ obtained from a large scale assay with methyl iodide. ${ }^{3 \mathrm{~b}}$ This provided a new product (6) that co-eluted with an authentic standard of dimethylstealthin C (Figures 3B and S15). Further HRMS analysis of intermediate 3a (Figure S14) showed a molecular weight $\mathrm{m} / z$ [M $+\mathrm{H}]^{+}=531.0898$ and fragmentation pattern consistent with appendage of a cysteine disulfide to the benzofluorene scaffold. 
Inclusion of other proteinogenic amino acids in $\mathrm{AlpJ} / \mathrm{Fre}$ in vitro assays did not provide new products (data not shown).

The production of $\mathbf{3}$ from $\mathbf{1}$ and L-cysteine by AlpJ and KinO1/Fre is interesting from a mechanistic perspective. To verify the origin of the amino substituent of $\mathbf{3}$, we performed the in vitro assay with ${ }^{15} \mathrm{~N}$-labeled L-cysteine (Figure S12, trace iv) and observed the masses of $\mathbf{3}$ and $\mathbf{3 a}$ increase by +1 and +2 mass units, respectively (Figures S18 and S19). As AlpJ could convert 1 to either 3 in the presence of L-cysteine or 2 in the presence of $N$ acetyl-L-cysteine, we deduced that the presence of a free amine might be a critical determinant of reaction outcome. We hypothesize that in a manner analogous to the formation of $\mathbf{2}$, generation of 3 involves attack by the L-cysteine thiol to form a thioether intermediate ( $N$-deacetylseongomycin, 7) followed by an intramolecular, S-N-type Smiles rearrangement (Scheme 1). ${ }^{15}$ This reactivity resembles the $\mathrm{S}$ - to $\mathrm{N}$-acyl transfer mechanisms of native chemical ligation and protein splicing. ${ }^{16}$ The resulting N-linked intermediate $(\mathbf{8})$ could form a disulfide bond with L-cysteine to afford 3a, which could be further oxidized to imine 9. Hydrolysis of this intermediate would provide 3 .

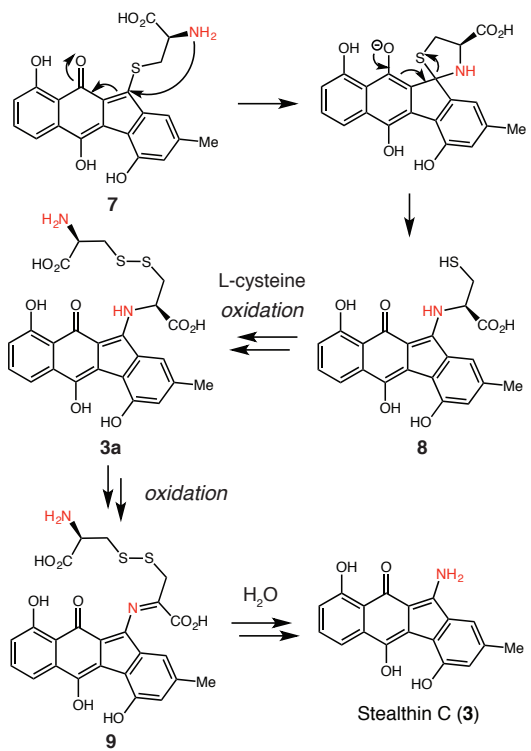

Scheme 1. Proposed mechanism for stealthin C formation.

To test this mechanistic proposal, we ran $\mathrm{AlpJ} /$ Fre in vitro assays with 1 and a panel of alternative amino acid co-substrates (Figure 4). L-serine and $S$-methyl-L-cysteine failed to provide new products, confirming the essential role of a thiol nucleophile (Figure S20, traces i and ii). L-cysteine analogs containing nonnucleophilic amino groups, including the dipeptide $\gamma$-Glu-Cys and $N$-carbamoyl-L-cysteine, generated new $S$-linked products (10 and 11). This result shows that a nucleophilic amino group is necessary for $\mathrm{C}-\mathrm{N}$ bond formation. (Figure S20, traces iii and iv, Figures $\mathrm{S} 21$ and S22). Assays with $N$-methyl-L-cysteine provided $N$ methyl-stealthin C (12) (Figure S20, trace v, Figure S23). We investigated the importance of the carboxylic acid of L-cysteine by examining cysteamine. HPLC and LC-HRMS analyses revealed a single new product $\left(\mathrm{m} / \mathrm{z}[\mathrm{M}-\mathrm{H}]^{-}=441.0949\right)$ that likely corresponds to mixed disulfide $\mathbf{1 3}$ (Figure S20, trace vi, and Figure S24). This result suggests that the carboxylic acid is not needed for the Smiles rearrangement but is critical for the final oxidation. Assays with L-homocysteine gave a single product with a mass corresponding to homocysteine adduct $14\left(\mathrm{~m} / \mathrm{z}[\mathrm{M}-\mathrm{H}]^{-}=\right.$ 424.0862) (Figure S20, trace vii and Figure S25). The failure to form 3 could reflect a reduced rate of formation for a six membered ring-containing intermediate. Finally, assays with Dcysteine generated $\mathbf{3}$ with efficiencies similar to that observed for L-cysteine (Figure S20, trace viii).
The broad reactivity observed with thiol nucleophiles and the lack of a preference for L- vs. D-cysteine led us to question whether AlpJ and/or Fre catalyzed the thiol addition and Smiles rearrangement reactions involved in forming $\mathbf{2}$ and $\mathbf{3}$. To examine this issue, we performed competition experiments in which AlpJ and Fre were incubated with $\mathbf{1}$ and equal concentrations of two thiols. Assays containing L-cysteine and $\mathrm{N}$-acetyl cysteine, as well as L-cysteine and cysteamine, generated comparable amounts of both expected products (Figure S20, trace ix). A third product with a mass corresponding to a mixed disulfide was also produced in the assay containing L-cysteine and cysteamine (Figure S20, trace $x$ and Figure S26). Overall, the failure to observe a strong preference for L-cysteine over other thiols suggests this reactivity is non-enzymatic. Conversion of $\mathbf{3 a}$ to $\mathbf{3}$ was observed when purified 3a was incubated alone, as well as after assay mixtures containing 3a were boiled to denature enzymes (Figure S27), indicating that the oxidation of $\mathbf{3 a}$ and imine hydrolysis are likely also non-enzymatic.
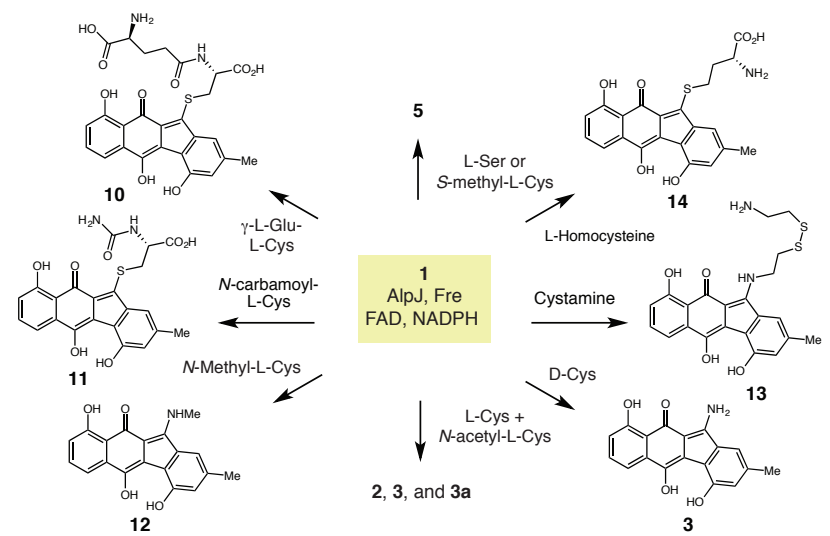

Figure 4. Reactivity of AlpJ-derived intermediates toward alternate nucleophiles.

The non-enzymatic generation of $\mathbf{3}$ from L-cysteine and an AlpJ-derived reaction partner in vitro has implications for the in vivo synthesis of this metabolite. 3 has only been isolated from mutant strains, ${ }^{6 \mathrm{~b}}$ consistent with non-enzymatic production from an accumulating, reactive species. This general phenomenon has been identified in other biosynthetic pathways. ${ }^{17}$ The relevance of 3 to kinamycin biosynthesis was established using feeding experiments, ${ }^{6 \mathrm{~b}}$ and this result led to the hypothesis that diazo group construction involves $\mathrm{N}-\mathrm{N}$ bond formation on the ben$z o[b]$ fluorene scaffold. However, the $\mathbf{3}$ used for these feeding experiments was carbon-labeled and only low levels of incorporation were observed. Though additional, unidentified enzymes could provide an alternate route to 3 in vivo, the observation that this metabolite is readily accessed via non-enzymatic reactions and the propensity of this ring system to undergo substitution chemistry led us to question the stability of $\mathbf{3}$ in the original feeding experiments and its role as a biosynthetic intermediate.

To investigate this possibility, we performed preliminary feeding experiments with the kinamycin producer $S$. murayamaensis. Feeding ${ }^{15} \mathrm{~N}$-labeled L-cysteine resulted in undetectable labeling of kinamycin $\mathrm{D}$, whereas feeding ${ }^{15} \mathrm{~N}_{4}$-labeled L-arginine as a control provided low but detectable incorporation (Table S3). The lack of kinamyin $\mathrm{D}$ enrichment upon feeding ${ }^{15} \mathrm{~N}$-labeled Lcysteine suggests that $\mathbf{3}$ arises from off-pathway reactions of a shunt product generated in mutants blocked in kinamycin synthesis. Significantly, this finding, along with recent investigations of hydrazide assembly in fosfazinomycin biosynthesis, ${ }^{18}$ raises serious doubts about the long-assumed intermediacy of $\mathbf{3}$ in this pathway and will reshape future efforts to decipher the enzymatic chemistry of diazo formation. 
Discovering new approaches for aromatic $\mathrm{C}-\mathrm{N}$ bond formation is important because of this structural motif's predominance in pharmaceuticals and other bioactive compounds. ${ }^{19}$ Interfacing the non-enzymatic S-N-type Smiles rearrangement with a natural product biosynthetic pathway represents a distinct strategy for accessing aromatic $\mathrm{C}-\mathrm{N}$ bond containing compounds. Our findings should therefore inspire related strategies in synthetic biology.

\section{ASSOCIATED CONTENT}

\section{Supporting Information}

Full experimental procedures and characterization data. This material is available free of charge via the Internet at http://pubs.acs.org.

\section{AUTHOR INFORMATION}

\section{Corresponding Author}

* Emily P. Balskus

balskus@chemistry.harvard.edu.

\section{Author Contributions}

The manuscript was written through contributions of all authors. All authors have given approval to the final version of the manuscript.

\section{Notes}

The authors declare no competing financial interests.

\section{ACKNOWLEDGMENTS}

We thank Prof. Sean F. Brady (The Rockefeller University) for the gift of BAC AB649/1850, Prof. Wenjun Zhang (University of California, Berkeley) for the gifts of $S$. lividans TK64 and pUL201PW, and Bristol-Myers Squibb for the strain Streptomyces lividans K4-114. We thank Prof. Philip J. Proteau (Oregon State University) for providing the dimethyl-stealthin $\mathrm{C}$ and kinamycin D standards and Jennifer Wang (Harvard University) for assistance with LC-MS analyses. We acknowledge financial support from Harvard University, the Searle Scholars Program, and the NIH (DP2 GM105434).

\section{REFERENCES}

(1) Herzon, S. B.; Woo, C. M. Nat. Prod. Rep. 2012, $29,87$.

(2) Colis, L. C.; Woo, C. M.; Hegan, D. C.; Li, Z.; Glazer, P. M.; Herzon, S. B. Nat. Chem. 2014, 6, 504.
(3) (a) Seaton, P. J.; Gould, S. J. J. Antibiot. (Tokyo) 1989, 42, 189. (b) Cone, M. C.; Seaton, P. J.; Halley, K. A.; Gould, S. J. J. Antibiot. (Tokyo) 1989, 42, 179. (c) Bunet, R.; Song, L.; Mendes, M. V.; Corre, C.; Hotel, L.; Rouhier, N.; Framboisier, X.; Leblond, P.; Challis, G. L.; Aigle, B. J. Bacteriol. 2011, 193, 1142.

(4) (a) Rix, U.; Wang, C.; Chen, Y.; Lipata, F. M.; Remsing Rix, L. L.; Greenwell, L. M.; Vining, L. C.; Yang, K.; Rohr, J. Chembiochem 2005, 6, 838. (b) Chen, Y. H.; Wang, C. C.; Greenwell, L.; Rix, U.; Hoffmeister, D.; Vining, L. C.; Rohr, J.; Yang, K. Q. J. Biol. Chem. 2005 , 280, 22508. (c) Fan, K.; Pan, G.; Peng, X.; Zheng, J.; Gao, W.; Wang, J.; Wang, W.; Li, Y.; Yang, K. Chem. Biol. 2012, 19, 1381. (d) Tibrewal, N.; Pahari, P.; Wang, G.; Kharel, M. K.; Morris, C.; Downey, T.; Hou, Y.; Bugni, T. S.; Rohr, J. J. Am. Chem. Soc. 2012, 134, 18181.

(5) Seaton, P. J.; Gould, S. J. J. Am. Chem. Soc. 1987, 109, 5282.

(6) (a) Gould, S. J.; Melville, C. R. Bioorg. Med. Chem. Lett. 1995, 5, 51. (b) Gould, S. J.; Melville, C. R.; Cone, M. C.; Chen, J.; Carney, J. R. J. Org. Chem. 1997, 62, 320. (c) Carney, J. R.; Hong, S. T.; Gould, S. J. Tetrahedron Lett. 1997, 38, 3139.

(7) Woo, C. M.; Gholap, S. L.; Herzon, S. B. J. Nat. Prod. 2013 76,1238 .

(8) Gould, S. J.; Hong, S. T.; Carney, J. R. J. Antibiot. (Tokyo) $1998,51,50$

(9) (a) Janso, J. E.; Haltli, B. A.; Eustaquio, A. S.; Kulowski, K.; Waldman, A. J.; Zha, L.; Nakamura, H.; Bernan, V. S.; He, H.; Carter, G. T.; Koehn, F. E.; Balskus, E. P. Tetrahedron 2014, 70, 4156. (b) Kersten, R. D.; Lane, A. L.; Nett, M.; Richter, T. K.; Duggan, B. M.; Dorrestein, P. C.; Moore, B. S. Chembiochem 2013, 14, 955.

(10) Wang, B.; Ren, J. W.; Li, L. Y.; Guo, F.; Pan, G. H.; Ai, G. M.; Aigle, B.; Fan, K. Q.; Yang, K. Q. Chem. Commun. 2015, 51, 8845.

(11) Kharel, M. K.; Pahari, P.; Shepherd, M. D.; Tibrewal, N.; Nybo, S. E.; Shaaban, K. A.; Rohr, J. Nat. Prod. Rep. 2012, 29, 264.

(12) Newton, G. L.; Arnold, K.; Price, M. S.; Sherrill, C.; Delcardayre, S. B.; Aharonowitz, Y.; Cohen, G.; Davies, J.; Fahey, R. C.; Davis, C. J. Bacteriol. 1996, 178, 1990.

(13) Spyrou, G.; Haggard-Ljungquist, E.; Krook, M.; Jornvall, H.; Nilsson, E.; Reichard, P. J. Bacteriol. 1991, 173, 3673.

(14) Gould, S. J.; Melville, C. R. Tetrahedron Lett. 1997, 38, 1473.

(15) Castagnolo, D.; Pagano, M.; Bernardini, M.; Botta, M. Tetrahedron Lett. 2012, 53, 5008.

(16) (a) Kane, P. M.; Yamashiro, C. T.; Wolczyk, D. F.; Neff, N.; Goebl, M.; Stevens, T. H. Science 1990, 250, 651. (b) Hirata, R.; Ohsumk, Y.; Nakano, A.; Kawasaki, H.; Suzuki, K.; Anraku, Y. J. Biol. Chem. 1990, 265, 6726 .

(17) (a) Rix, U.; Zheng, J.; Remsing Rix, L. L.; Greenwell, L.; Yang, K.; Rohr, J. J. Am. Chem. Soc. 2004, 126, 4496. (b) Yan, Y.; Yang, J.; Yu, Z.; Yu, M.; Ma, Y.; Wang, L.; Su, C.; Luo, J.; Horsman, G. P.; Huang, S. Nat. Commun. 2016, 7, 13083. (c) Hu, Y.; Potts, M. B.; Colosimo, D.; Herrera-Herrera, M. L.; Legako, A. G.; Yousufuddin, M.; White, M. A.; MacMillan, J. B. J. Am. Chem. Soc. 2013, 135, 13387.

(18) Huang, Z.; Wang, K. K.; van der Donk, W. A. Chem. Sci. 2016, 7, 5219 .

(19) Hili, R.; Yudin, A. K. Nat. Chem. Biol. 2006, 2, 284. 
TOC graphic:

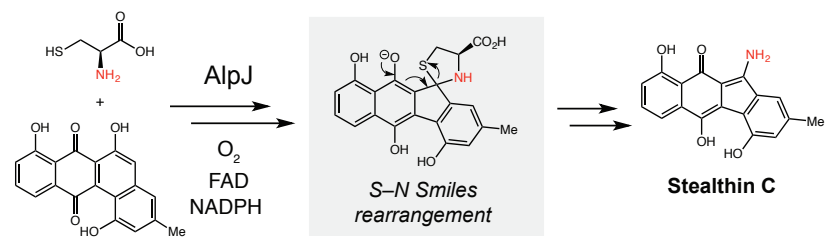

